\title{
Serum metabolomics study of end-stage renal disease with depression: potential biomarkers for diagnosis and promising targets for therapy
}

Jie Hu

Laboratory of Stem Cells and Tissue Engineering,Department of Histology and Embryology,Chongqing Medical University

\section{Dezhi Yuan}

Department of Neurology,the Second Affiliated Hospital of Chongqing Medical University

\section{Kuan Tian}

Laboratory of Stem Cells and Tissue Engineering,Department of Histology and Embryology,Chongqing Medical University

\section{Liping Feng}

Department of Neurology,the Second Affiliated Hospital of Chongqing Medical University

\section{Qiuye Zhao}

Department of Neurology,the Second Affiliated Hospital of Chongqing Medical University

\section{Xiaohui Liao}

Department of Nephrology,the Second Affiliated Hospital of Chongqing Medical University

Huirong Luo

Laboratory of Stem Cells and Tissue Engineering,Department of Histology and Embryology,Chongqing Medical University

\section{Qihui Jiang}

Department of Neurology,the Second Affiliated Hospital of Chongqing Medical University

\section{Xuefei Wang}

Department of Neurology,the Second Affiliated Hospital of Chongqing Medical University

\section{Xiaotian Zhang}

Department of Neurology,the Second Affiliated Hospital of Chongqing Medical University

\section{Ling Hu}

Laboratory of Stem Cells and Tissue Engineering,Department of Histology and Embryology,Chongqing Medical University

\section{Hongkai Wang}

Graduate Student Interdepartmental Neuroscience Program (NUIN) Northwestern University

\section{Peng Zhou}

Laboratory of Stem Cells and Tissue Engineering,Department of Histology and Embryology,Chongqing Medical University 


\section{Xingxian Guo}

Laboratory of Stem Cells and Tissue Engineering,Department of Histology and Embryology,Chongqing Medical University

Jianhua Ran

Laboratory of Stem cells and Tissue Engineering,Department of Histology and Embryology,Chongqing Medical University

Jinfang Li ( $\sim$ lijinfang@hospital.cqmu.edu.cn )

The Secound Affiliated Hospital of Chongqing Medical University https://orcid.org/0000-0001-69712602

\section{Research article}

Keywords: End-stage renal disease; depression; metabolomics; UPLC-QTOF-MS; biomarkers

Posted Date: November 6th, 2019

DOI: https://doi.org/10.21203/rs.2.16938/v1

License: (c) (1) This work is licensed under a Creative Commons Attribution 4.0 International License. Read Full License 


\section{Abstract}

Background End-stage renal disease (ESRD) is the most severe stage during the development of the renal failure. And depression is the most common psychological disorder in patients with ESRD, which in turn aggravates the progression of renal failure and seriously reduce the quality of life in ESRD patients with depression, but its underlying mechanism remains unclear. This study aimed to reveal the pathogenesis and discover novel peripheral biomarkers for ESRD with depression through metabolomics analysis.Methods Ultra-high-performance liquid chromatography tandem quadrupole time-of-flight mass spectrometry (UPLC-QTOF-MS) was used to explore changes of serum metabolites among healthy controls $(n=12)$, ESRD patients $(n=17)$, and ESRD patients with depression $(n=17)$. Also, the differential metabolites between groups were subjected to clustering analysis, pathway analysis, receiver operating characteristic (ROC) curve analysis.Results A total of 57 significant serum differential metabolites were identified between the ESRD without depression group and the ESRD with depression group, which were involved in 19 metabolic pathways, such as energy metabolism, glycerolipid metabolism and glutamate-centered metabolism. Moreover, the area under the ROC curve of Gentisic acid, Uric acid, 5-HT, 2-Phosphoglyceric acid, Leucyl-phenylalanine, Propenoyl carnitine, Malaoxon, Pregnenolone, 6-Thioxanthine 5'-monophosphate, Hydroxyl ansoprazole, Zileuton 0-glucuronide, Cabergoline, PA (16:0/18:2(9Z,12Z)), PG (18:0/18:1(11Z)), probucol, etc. and their combination was greater than 0.90 .Conclusions Inflammation, oxidative stress and metabolic abnormalities in energy metabolism, glycerolipid metabolism and glutamate-centered metabolism may be associated with the pathogenesis of ESRD with depression, which may be promising targets for therapy. Furthermore, the identified differential metabolites may serve as biomarkers for the diagnosis of ESRD patients with depression.

\section{Background}

End-stage renal disease (ESRD) is the most sever stage of the acute and chronic renal failure, with massively accumulated metabolic end products and toxic substances, disordered electrolytes and acidbase balance, as well as some endocrine dysfunction, resulting in a series of autotoxic symptoms [1]. In patients with ESRD, depression is the most common psychological disorder, with a prevalence rate as high as $20 \%-25 \%$ by some contemporary estimates [2,3]. Depression has been identified as a complicating comorbid diagnosis in ESRD, shown as low mood, slow thinking, cognitive impairment, physical symptoms and even suicide [3-5]. There is overwhelming evidence that chronic illness with comorbid depression is associated with increased symptom burden and functional impairment, poor quality of life, non-adherence to treatment, and worse clinical outcomes [6-8]. Nevertheless, the pathogenesis of ESRD with depression remains unclear.

The kidney is a metabolically-active organ involved in the handling of biochemical classes of metabolites [9]. One of the hallmarks of progression to ESRD is the plasma accumulation of certain metabolites, uremic solutes $[10,11]$. Therefore, the metabolomic profiling of ESRD patients may be a promising method to identify new biomarkers for the prognoses of ESRD patients. Ultra-performance liquid 
chromatography tandem mass spectrometry (UPLC-MS) has high selectivity, high sensitivity and good time-retention reproducibility, thereby suitable for metabolome analysis, especially for non-targeted metabolome studies [12]. Therefore, recently, metabolomics on chronic kidney disease (chronic kidney disease, CKD) has become a global hotspot. Previous studies[9] have found that there are significant statistical differences between the levels of various metabolic products of the CKD group. While other studies have revealed that the levels of atty acid metabolism, particularly the polyunsaturated fatty acids (PUFAs) metabolism, and purine metabolism, are significantly different between depressed and nondepressed patients[13]. However, there is no metabolomic study focusing on ESRD with depression to our best knowledge. However, there is no metabolomic study focusing on ESRD with depression to our best knowledge.

In this study, we aimed to perform a metabolomics analysis to discover novel peripheral biomarkers for ESRD with depression as well as to elucidate the underlying mechanisms that initiate this progression. Our results may provide new theoretical basis for the complicated metabolic disorder and pathogenesis of ESRD with depression.

\section{Methods}

\section{Study populations}

All participants had signed written informed consent papers before study. The procedures of this study were approved by Ethics Committee of Chongqing Medical University. From January 2016 to July 2017, 17 ESRD patients, and 17 ESRD patients with depression in Department of Nephrology in Second Affiliated Hospital of Chongqing Medical University were enrolled in this study. The determination of depression was according to Hamilton Depression Rating Scale for Depression (HAMD) [39]: depression group with total score $>17$ points, and non-depression group with total score $\leq 17$ points. Additionally, 12 healthy subjects with matched age, gender, and body mass index (BMI) recruited from physical examination center were included as control group.

Inclusion criteria were as follows: (1) Confirmed as chronic renal failure uremia; (2) Serum creatinine > $707 \mathrm{mmol} / \mathrm{L}$ and endogenous creatinine clearance $<15 \mathrm{ml} /(\mathrm{min} \cdot 1.73 \mathrm{~m} 2)$; (3) The diagnosis and treatment of the disease were informed; (4) With normal liver function and blood glucose fluctuation range of 5.4 to $11.2 \mathrm{mmol} / \mathrm{L}$; (5) Brain computed tomography and magnetic resonance imaging showed no new lesions; (6) Without previous history of drug poisoning and psychosis. Patients with severe complications or severely impaired organs except for kidney were excluded.

\section{Sample and clinical data collection}

All subjects underwent venous blood collection $10 \mathrm{~h}$ after fasting. Collected blood was stored at $4{ }^{\circ} \mathrm{C}$ for 30-60 min followed by centrifugation at $3000 \mathrm{~g} / \mathrm{min}$ for $10 \mathrm{~min}$, and the supernatant was stored. The samples were stored at $-80^{\circ} \mathrm{C}$ with drikold as cold chain during transport. All subjects underwent routine 
(height, weight, blood pressure, and BMI) and biochemical examination. Plasma biochemical indicators for subjects are shown in Table 1.

\section{Sample preparation}

Before analysis, every frozen plasma sample was thawed and dissolved at $4^{\circ} \mathrm{C}$. A mixture of acetonitrile/methanol $(75: 25 \mathrm{v} / \mathrm{v}, 300 \mu \mathrm{l})$ (Merck, Germany) was added to plasma (100 $\mu \mathrm{l})$ to precipitate proteins. After vortexing for $60 \mathrm{~s}$, the mixture was stood for $10 \mathrm{~min}$ and then centrifuged at $12000 \mathrm{~g} / \mathrm{min}$ for $10 \mathrm{~min}$ at $4^{\circ} \mathrm{C}$. The supernatant was filtered through $0.22 \mu \mathrm{m}$ syringe filters (Jinteng, China) and then analyzed by UPLC-MS. Samples were subjected to quality control (QC) by pooling equal volumes of different individual serum samples to assess the reproducibility and reliability of the UPLC-MS system. QC of mixed samples was interspersed at the start, middle, and end of the test.

\section{Liquid chromatography-mass spectrometry (LC-MS) analysis}

Liquid chromatography (LC) separation was performed on ZORBAX Eclipse Plus C18 column $(2.1 \times 100$ $\mathrm{mm}, 3.5 \mu \mathrm{m}$; Agilent, USA). The column was maintained at $45^{\circ} \mathrm{C}$. A $10 \mu \mathrm{l}$ sample was injected into the column for each run in the full loop injection mode. The flow rate of the mobile phase was $0.5 \mathrm{ml} / \mathrm{min}$. In reversed-phase liquid chromatographic (RPLC) mode, gradient elution was performed with the following solvent system: (A) $0.1 \%$ formic acid-water (Merck, Germany), (B) acetonitrile with $0.1 \%$ formic acid. The gradient started with $98 \% \mathrm{~A}$ and decreased to $10 \% \mathrm{~A}$ in $13 \mathrm{~min}$, holding at $10 \% \mathrm{~A}$ for $3 \mathrm{~min}$, and then turned to $98 \%$ A immediately, holding at $98 \%$ A for 4 min. Mass spectrometry experiments were performed on Triple TOF $5600+$, an orthogonal accelerated time of flight mass spectrometer (AB SCIEX, USA) equipped with an electrospray ion source. Data were acquired in positive and negative-V-geometry mode for each chromatography separation technique LC-MS analysis. The mass spectrum parameters were as follows: the capillary voltages $2500 \mathrm{~V}$ and $3000 \mathrm{~V}$, cone gas $50 \mathrm{~L} / \mathrm{h}$, desolvation gas $600 \mathrm{~L} / \mathrm{h}$, source temperature $120^{\circ} \mathrm{C}$ and desolvation temperature $500^{\circ} \mathrm{C}$. The scan range was from 50 to $1500 \mathrm{~m} / \mathrm{z}$ in the full scan mode and data were collected in centroid mode. Data were centralized during acquisition using independent reference lock-mass ions via the Analyst TF 1.6 and Marker View 1.2.1.

Metabolites were identified by searching the free databases of Human Metabolome Database (HMDB) (http://www.hmdb.-ca/spectra/ms/search) [40]. The mass tolerance for the HMDB database search was set at $0.05 \mathrm{Da}$. The chromatographic retention behavior was used to reduce false-positive matches.

\section{Statistical analysis}

The multivariate analyses, including unsupervised principal component analysis (PCA), supervised partial least squares discriminant analysis (PLS-DA) and orthogonal projections to latent structures discriminant analysis (OPLS-DA), were conducted to determine the distributions and identify the metabolic difference in two or three groups using the MetaboAnalyst 4.0 (http://www.metaboanalyst.ca /MetaboAnalyst/) [41, 42]. The parameter R2 was used to evaluate the fitting condition of the PLS-DA models, and Q2 was used to assess the predictive ability. These parameters ranged from 0 to 1 , where 1 indicated a perfect fit. 
When the values of R2 and Q2 were > 0.5, the model considered to be successful. To avoid overfitting, 7fold cross-validation and response permutation testing (RPT) were used for model validation[43]. In the PLS-DA model, variables with variable important in projection (VIP) $>1$ considered to be potentially differential metabolites. Meanwhile, single variable statistical analysis was performed on the identified metabolites.

Welch's t-test was used to compare the two groups that were correlated with the intensities of the integrated regions using MetaboAnalyst 4.0 , and $p$-value $<0.05$ was considered statistically significant. Moreover, peaks with consistently upregulated or downregulated were identified, the regional intensity data of which were used in hierarchical cluster analysis and metabolic pathway analysis.

\section{Pathway analysis}

The differential chemical metabolites were subjected to pathway analysis with Metaboanalyst web portal (http://www.metaboanalyst.ca/) followed by visualization. Additional powerful pathway enrichment analysis was conducted by Metabolite Set Enrichment Analysis (MSEA)

(http://www.msea.ca/MSEA/faces/Home.jsp). Pearson's $r$ correlation was calculated to evaluate the relations among the biomarkers $(p<0.05$, impact $>0.01)$.

\section{Receiver operating characteristic (ROC) curve analysis}

ROC curve was used to investigate the diagnostic value of differential metabolites. The area under the ROC curve (AUC) indicates the overall ability of the test. A test with an AUC greater than 0.9 has high accuracy, while $0.7-0.9$ indicates moderate accuracy, $0.5-0.7$, low accuracy and 0.5 a chance result. ROC curve was obtained using the SPSS 25.0 software.

\section{Results}

\section{Biochemical characteristics}

The demographics and clinical characteristics of the subjects are shown in Table 1. There was no significant difference in age, gender and BMI between the ESRD patients (with or without depression) and the healthy controls. Additionally, compared with the control group, the Neutrophilic granulocyte\% (N\%), Systolic blood pressure (SBP), Diastolic blood pressure (DBP), Triglyceride (TG), Creatinine (Cr), Blood urea nitrogen (BUN), Potassium ion $\left(\mathrm{K}^{+}\right)$, phosphorus and depression scores were significantly higher $(p<$ 0.05), while Total cholesterol (TC), HemoglobinखHB囚and albumin were lower $(p<0.05)$ in the ESRD group (with or without depression). Moreover, compared with the ESRD without depression group, the N\%, anxiety score and depression score were significantly higher in the ESRD with depression group $(p<$ 0.05). 
The distribution of samples, rationality of the experimental design, and homogeneity of biological replicates were determined by PCA. As shown in the score plot in the positive or negative ion mode, the ESRD and control groups were separated, but the ESRD without depression group and with depression group were not separated (Figure S1).

To improve the separation of the three groups, PLS-DA and OPLS-DA were performed to visualize their metabolic differences. The score plots of PLS-DA are shown in Figure 1. After response replacement test of these score plots, there was no overfitting, indicating that the PLS-DA model was successfully constructed. Moreover, the three groups were well separated in the OPLS-DA score plot, in both positive and negative ions (Figure 2). All models were cross-validated and no overfitting was identified. These results indicated that the ESRD patients and ESRD patients with depression had unique metabolic characteristics, making them to be separated.

\section{Differential metabolite analysis and identification}

According to the VIP values of characteristic variables obtained from the cross-validated OPLS-DA model, the potential markers were further screened. Variables with $\mathrm{FC}>5$ or $\mathrm{FC}<0.1, \mathrm{VIP}>1$ and $p<0.05$ were considered as potential markers and were structurally identified. A total of 643 characteristic compounds that were differential between ESRD and healthy control groups, 459 ones in ESRD with depression group vs. healthy control group, 57 in ESRD without depression group vs. ESRD with depression group were identified. The screened differential metabolites were mapped to HMDB to identify specific substances. Qualitative results and related information of differential metabolites were screened in positive and negative ion modes. Results of ESRD without depression group vs. ESRD with depression group are shown in Table 2.

\section{Clustering analysis}

Bidirectional clustering of samples and metabolites was performed on all metabolites using the hierarchical clustering analysis. The heatmaps of the differential metabolites in each sample are shown in Figure 4, which provide an intuitive understanding of the relative content of each metabolite.

\section{Pathway analysis}

Pathway analysis of differential compounds was performed using the KEGG database. The relevant influence scores $(-\log (p)>0.5$, impact $>0.01)$ of metabolic pathways enriched by the differential compound in ESRD without depression group vs. ESRD with depression group are shown in Table 3. The metabolic pathways of ESRD without depression group vs. healthy controls shown in Table S1. For simplicity, the metabolic pathways were converted into a pathway overview map, each point representing a pathway (Figure 5). There were 19 differential serum metabolite pathways in the ESRD without depression group vs. ESRD with depression group, and 16 of them were key pathways. The metabolic 
pathway overview maps of ESRD without depression group vs. healthy control group are shown in Figure S2.

\section{ROC analysis}

To obtain a simple metabolite combination that can separate ESRD and ESRD with depression in clinical practice, we further analyzed the 57 differential metabolites in the main metabolic pathway. To investigate the diagnostic value of these differential metabolites, ROC curve analysis was conducted to assess the sensitivity and specificity of these metabolites. The compounds with AUC $>0.90$ are shown in Table 4.

\section{Discussion}

This study examined serum metabolite differences between ESRD/ ESRD with depression and healthy controls, and between ESRD and ESRD with depression. Based on the pattern recognition method and the recognition model (PLS-DA, OPLS-DA), metabolite changes between groups were distinguished, and satisfactory model parameters were obtained. Through multivariate and univariate statistical analyses, the unique metabolic patterns related to ESRD were also obtained. The differential metabolites in ESRD group and healthy controls were involved in metabolic pathways such as alanine, aspartate and glutamate metabolism, phenylalanine metabolism, glutathione metabolism, and cysteine and methionine metabolism. ESRD with depression group was significantly different from ESRD without depression group in metabolic pathways, such as energy metabolism, glycerolipid metabolism and glutamatecentered metabolism (Figure 6). Additionally, differential metabolites with high diagnostic performance may serve as potential diagnostic markers for distinguishing ESRD and ESRD with depression patients.

\section{Metabolic disorders in ESRD patients}

\section{Clinical biochemical characteristics of ESRD patients}

Compared with the healthy control group, the SBP, DBP, TG, Cr, BUN, $\mathrm{K}^{+}$, phosphorus and depression scores of the ESRD without depression group were increased, while HB and albumin were significantly decreased. These biochemical changes are consistent with hypertension, azotemia, and anemia in ESRD patients, and suggest a high inflammatory response in ESRD patients.

\section{Reduction of antioxidants}

Glutathione, taurine and hypotaurine metabolism, cysteine and methionine metabolic pathways are abnormal in ESRD patients, and metabolites in these pathways such as: S-adenosyl methionine (SAM), glutathione (GSH) and taurocholic acid, etc. antioxidants were lower than that of the healthy control group. The GSH structure contains an active sulfhydryl group (-SH) that is easily oxo-dehydrogenated; studies have shown that SAM inhibits the strong inflammatory and oxidative stress processes that occur 
in patients[14, 15]. Therefore, ESRD patients have reduced antioxidant capacity and may have oxidative stress damage in vivo, which is consistent with the findings of Kalender B et al. [16].

\section{Metabolic disorders of aromatic amino acids}

Phenylalanine, tyrosine and tryptophan belong to aromatic amino acids, in which phenylalanine is catalyzed by phenylalanine hydroxylase to form tyrosine, and tyrosine is further metabolized to produce catecholamine (dopamine, norepinephrine and adrenaline). Compared with the healthy control group, the tyrosine content of the ESRD without depression group was decreased, which was consistent with previous studies[17, 18], and the decrease of tyrosine was also observed in patients with renal failure and diabetes[19]. In addition, patients with ESRD had a lower Kynurenine (KYN) and a higher 3Hydroxyanthranilic acid (3-HANA) than the healthy controls. Tryptophan is mainly metabolized by the kynurenine pathway (KP) and the 5-TH metabolic pathway, the former being more than $95 \%$ in mammals; $\mathrm{KYN}$ can inhibit antigen presentation, suppress immune response, and ultimately reduce inflammation[20]. However, 3-HANA is neurotoxic and induces the formation of free radicals such as hydroxyl radicals and hydrogen peroxide, and raises the level of oxidative stress[21]. It is concluded that patients with ESRD may be in a state of high inflammatory response and oxidative stress.

\section{ESRD with metabolic disorders in patients with depression}

\section{Clinical biochemical characteristics of ESRD patients with depression}

In our study, the neutrophil percentage of the ESRD with depression group was higher than that of the ESRD without depression group $(p<0.05)$. The occurrence of depression is highly related to inflammation[22], which is shown obviously in ESRD patients, with neutrophils as the indicator of inflammatory response[23]. Turkmen et al.[24] reported that the presence of inflammatory factors (such as TNF-a, IL-4, IL-6) in ESRD patients may be effectively regulated by the hypothalamic-pituitary-adrenal axis (HPA); Inflammatory factors can also directly stimulate HPA to cause abnormalities. Studies have shown that HPA abnormalities are one of the main causes of depression. Therefore, high inflammatory response in ESRD patients with depression may be the pathological basis of depression.

\section{Energy metabolism}

Compared with the ESRD without depression group, the ESRD with depression group had higher N-acetylL-aspartic acid (NAA) and gentisic acid, and lower 5-HT and thiamine pyrophosphate (TPP). KEGG analysis showed that these metabolites were involved in the tricarboxylic acid cycle (TCA cycle). TCA cycle is the ultimate and hub metabolic pathway of three major nutrients (sugars, lipids and amino acids). TCA metabolic abnormality has been reported in ESRD with depression[13]. Disorder of nutrient metabolism is common in ESRD patients, leading to insufficient energy supply for biochemical reactions. 
NAA as a biomarker of neuronal damage severity, only exists in neurons, which is one of the most concentrated metabolites in the human brain, and is not detected in the blood[25,26]. This study revealed that the NAA level in the ESRD with depression group was higher than that in the ESRD without depression group, which may be due to neuronal apoptosis and necrosis, indicating that neuronal activity was reduced or functional damage in ESRD patients with depression. 5-HT is an important neurotransmitter, and the lack of $5-\mathrm{HT}$ in the central nervous system can result in depression. Reduction of 5-HT function and activity is closely related to depression, loss of appetite, and endocrine dysfunction[27]. This phenomenon can be observed in patients with major depression [29]. In accordance with the findings above, our result showed that the 5-HT level in ESRD patients with depression was lower than that in ESRD patients. We speculated that the factors affecting the metabolism of tryptophan to 5$\mathrm{HT}$ and further metabolism to melatonin or acetyl-CoA in ESRD with depression patients may be one of the causes of depression.

$\mathrm{KP}$ in the inflammatory hypothesis of depression [30]: the emergence of inflammatory depression is caused by immune function and neurotransmitter changes in the activation of Indoleamine 2,3dioxygenase (IDO), which not only leads to tyrosine failure also causes an increase in neurotoxic products through KP, resulting depression. There have been KP disorders in ESRD patients in this study, which may be one of the causes of depression with the prolongation of ESRD.

Thiamine, the vitamin B1, whose biologically active form is TPP[28]. TPP is an important cofactor for pyruvate dehydrogenase complex (PDHC), a-ketoglutarate dehydrogenase complex (KGDHC) and branched-chain a-keto acid dehydrogenase (BCKDH) [29]. PDHC and KGDHC are important components of cells using glucose to produce adenosine triphosphate (ATP) pathways, and BCKDH is a key enzyme for gluconeogenesis [30]. Consequently, thiamine plays an important role in maintaining the balance of oxidative metabolism in the brain. In patients with ESRD, the accumulation of toxins results in gastrointestinal reactions such as nausea, vomiting, and loss of appetite, leading to insufficient intake of thiamine, meanwhile, water-soluble thiamine is lost during dialysis, which together lead to thiamine deficiency. Deficiency of TPP can lead to metabolic disorders of sugar, lipids and amino acids, resulting in the reduction of ATP synthesis in the brain; moreover, TPP deficiency leads to cytotoxic edema, exacerbation of excitotoxic damage, oxidative stress damage, induction of inflammatory reaction, and destruction of blood brain barrier; all above considered to be potential mechanisms of depression [30, 31]. Specially, TPP catalyzes the conversion of tryptamine to 5-HT by increasing the activity of decarboxylation enzyme, thereby affecting the secretion of 5-HT[32]. In this study, ESRD patients with depression presented simultaneous descending of 5-HT and TPP, which may jointly promote the occurrence and deterioration of depression. Therefore, we speculated that serotonin reuptake inhibitors combined with thiamine may have a good anti-depressant effect on ESRD patients with depression.

\section{Glycerolipid metabolism}

In this study, we also found abnormalities in the glycerolipid metabolism, with elevated LysoPC (18:1 (9Z)), PG (18:0/18:1 (11Z)) and PA (16:0/18: 2 (9Z, 12Z)) in ESRD with depression compared to the ESRD 
without depression group. Phospholipids that account for $60 \%$ of the brain weight, is critical for brain neuronal structures, especially synaptic structures. The three phospholipids of PA, PG and LysoPC play important roles in signal transduction of dopamine, serotonin, glutamate and acetylcholine[33]. Study has reported that PA, PG and LysoPC are important signaling molecules with various biological functions involved in cell proliferation and inflammatory processes[34], but the specific mechanism remains to be further studied.

\section{Glutamate-centered metabolism}

The glutamate-centered metabolism was also a main pathway enriched by the differential metabolites between the ESRD with depression and ESRD without depression groups, such as L-glutamic acid (decreased in the ESRD with depression group), urocanic acid and creatine (elevated in the ESRD with depression group). Glutamate is an excitatory neurotransmitter with the highest content, the widest distribution and the strongest effect in central nervous system[35]. Glutamate can be recycled in brain cells by two conversion mechanisms. First, glutamate is the starting material for GABA biosynthesis, catalyzed by Glutamic acid decarboxylase (GAD), which is thought to be associated with mood disorders and schizophrenia[36]. In addition, glutamate is also the starting material for the synthesis of glutamine by Glutamine synthetase (GS) in astrocytes and neutralizes ammonia during biochemical metabolism[37]. Cryan et al.[38] have reported the reduction in glutamate-glutamine cycle in plasma and cerebrospinal fluid in ESRD patients with depression. In the present study, L-glutamate deficiency may be one of the causes of depression in patients. L-arginine is a downstream metabolite of L-glutamic acid as well as the sole provider of guanyl that in turn synthesizes creatine. Creatine can rapidly re-synthesize ATP for energy supply. In this study, the creatine may be increase in response to energy metabolism disorder.

\section{Biomarkers for diagnosis}

Based on the ROC curve analysis, 20 differential compounds have high diagnostic value (AUC $\geq 0.9$ ) and can be used as a biomarker for predicting ESRD with depression. Besides, the combined analysis of biomarkers with high diagnostic potency obtained an ROC curve (AUC $=0.945)$, which may be of diagnostic value superior to a single differential compound.

\section{Limitations}

There are some limitations in this study. First, this is a cross-sectional study with a relatively small sample size which is insufficient to fully reflect all metabolic changes in ESRD with depression patiants. Additionally, other biological samples, such as urine and cerebrospinal fluid are not tested to obtain more differential metabolites. Therefore, a cohort study with a larger sample size is needed to further confirm the findings and reveal the pathogenesis of ESRD and ESRD with depression.

\section{Conclusions}


Our study applied non-targeted metabolomics methods to study the metabolic characteristics of ESRD and ESRD with depression. Inflammation, oxidative stress and metabolic abnormalities in energy metabolism, glycerolipid metabolism and glutamate-centered metabolism may be associated with the pathogenesis of ESRD with depression, which may be promising targets for therapy. Furthermore, several novel biomarkers were identified, which can distinguish ESRD patients with or without depression.

\section{Abbreviations}

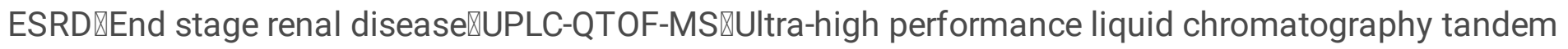

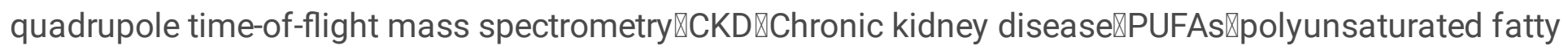

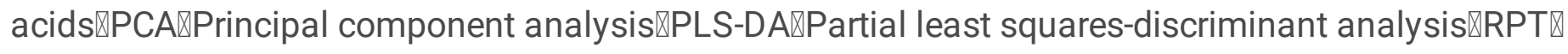

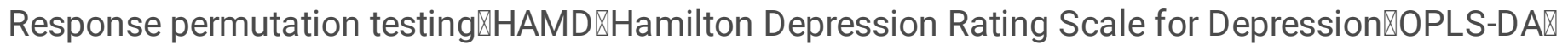
Orthogonal partial least square-discriminate analysis $₫ \mathrm{VIP} \otimes \mathrm{V}$ ariable important in projection $\mathbb{R O C} \quad \mathbb{}$

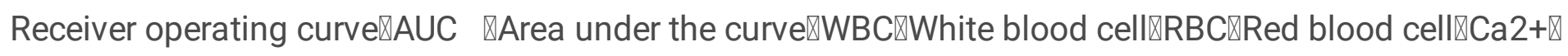

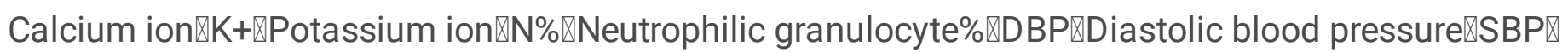

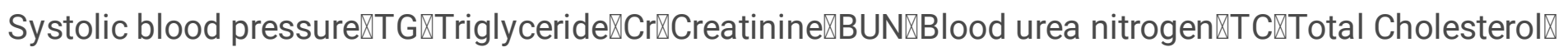

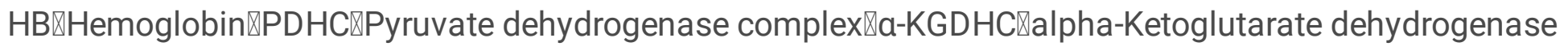
complex $\mathbb{B C K D H \otimes B r a n c h e d - c h a i n ~ a l p h a - k e t o ~ a c i d ~ d e h y d r o g e n a s e ~ c o m p l e x \bigotimes G A D \bowtie G l u t a m i c ~ a c i d ~}$ decarboxylase $\triangle H M D B \otimes T h e$ Human Metabolome Database $\triangle F C \otimes F o l d ~ c h a n g e \triangle K E G G \triangle K y o t o$ Encyclopedia

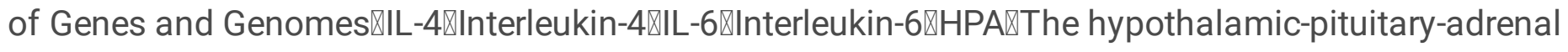

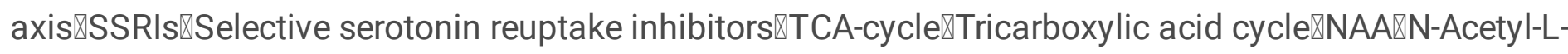

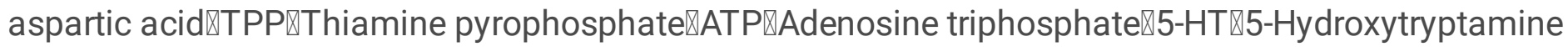

\section{Declarations}

\section{Acknowledgments}

We gratefully acknowledge the assistance of Department of Nephrology in the Second Affiliated Hospital of Chongqing Medical University and our colleagues Chunhua Fan, Yi Chen and Yanwei Lv during the writing of this paper and the helpful advice of Jing Li and Xin Tian.

\section{Authors' Contributions}

Ran J.H. and Li J.F. as well as Hu J. and Yuan D.Z. contributed to study design. Hu J., Yuan D.Z. and Tian K. contributed to data analysis and writing the paper. Zhao Q.Y., Wang H.K., Feng L.P., Liao X.H., Jiang Q.H., Wang X.F., Zhang X.T., Hu L., Luo H.R., Zhou P., Guo X.X.

\section{Funding}


This research was supported by National Natural Science Foundation of China (No.81770738, Ran J.H.) and Chongqing Municipal Health Bureau (No.2012-1-38, Li J.F.).

\section{Availability of data and materials}

The study data can be accessed from the corresponding author Ran J.H. or Li J.F. by request.

\section{Ethics approval and consent to participate}

The study protocol was approved by the Second Affiliated Hospital of Chongqing Medical University (Approved ID: 2019-Research No. 279-01).

\section{Consent for publication}

Not applicable.

\section{Competing interests}

The authors declare that they have no competing interest.

\section{References}

1. O'Shaughnessy, M. M.; Liu, S.; Montez-Rath, M. E.; Lafayette, R. A.; Winkelmayer, W. C., Cause of kidney disease and cardiovascular events in a national cohort of US patients with end-stage renal disease on dialysis: a retrospective analysis. Eur Heart $J$ 2019, 40, (11), 887-898.

2. Kimmel, P. L.; Peterson, R. A., Depression in patients with end-stage renal disease treated with dialysis: has the time to treat arrived? Clin J Am Soc Nephro/ 2006, 1, (3), 349-52.

3. American; Psychiatric; Association, Diagnostic and statistical manual of mental disorders. 5th ed. Washington, DC: APA 2013.

4. Biyik, Z.; Solak, Y.; Atalay, H.; Gaipov, A.; Guney, F.; Turk, S., Gabapentin versus pregabalin in improving sleep quality and depression in hemodialysis patients with peripheral neuropathy: a randomized prospective crossover trial. Int Urol Nephrol 2013, 45, (3), 831-7.

5. Tsai, Y. C.; Chiu, Y. W.; Hung, C. C.; Hwang, S. J.; Tsai, J. C.; Wang, S. L.; Lin, M. Y.; Chen, H. C., Association of symptoms of depression with progression of CKD. Am J Kidney Dis 2012, 60, (1), 5461. 
6. van Dooren, F. E.; Nefs, G.; Schram, M. T.; Verhey, F. R.; Denollet, J.; Pouwer, F., Depression and risk of mortality in people with diabetes mellitus: a systematic review and meta-analysis. Plos One 2013, 8 , (3), e57058.

7. Molnar, M. Z.; Streja, E.; Sumida, K.; Soohoo, M.; Ravel, V. A.; Gaipov, A.; Potukuchi, P. K.; Thomas, F.; Rhee, C. M.; Lu, J. L.; Kalantar-Zadeh, K.; Kovesdy, C. P., Pre-ESRD Depression and Post-ESRD Mortality in Patients with Advanced CKD Transitioning to Dialysis. Clin J Am Soc Nephro/ 2017, 12, (9), 1428-1437.

8. Greenwood, S. A.; Castle, E.; Lindup, H.; Mayes, J.; Waite, I.; Grant, D.; Mangahis, E.; Crabb, O.; Shevket, K.; Macdougall, I. C.; MacLaughlin, H. L., Mortality and morbidity following exercise-based renal rehabilitation in patients with chronic kidney disease: the effect of programme completion and change in exercise capacity. Nephrol Dial Transplant 2018.

9. Hocher, B.; Adamski, J., Metabolomics for clinical use and research in chronic kidney disease. Nat Rev Nephrol 2017, 13, (5), 269-284.

10. Flore, D.; Gerald, C.; Rita, D. S.; Mariano, R.; Joachim, J.; Raymond, V.; Angel, A., Normal and pathologic concentrations of uremic toxins. Journal of the American Society of Nephrology 2012, 23, (7), 1258-1270.

11. Rhee, E. P.; Souza, A.; Farrell, L.; Pollak, M. R.; Lewis, G. D.; Steele, D. J.; Thadhani, R.; Clish, C. B.; Greka, A.; Gerszten, R. E., Metabolite profiling identifies markers of uremia. Journal of the American Society of Nephrology 2010, 21, (6), 1041-2051.

12. Zhao, Y. Y.; Cheng, X. L.; Wei, F.; Xiao, X. Y.; Sun, W. J.; Zhang, Y.; Lin, R. C., Serum metabonomics study of adenine-induced chronic renal failure in rats by ultra performance liquid chromatography coupled with quadrupole time-of-flight mass spectrometry. 1.976 2012, 17, (1), 48-55.

13. Zhou, X.; Liu, L.; Lan, X.; Cohen, D.; Zhang, Y.; Ravindran, A. V.; Yuan, S.; Zheng, P.; Coghill, D.; Yang, L., Polyunsaturated fatty acids metabolism, purine metabolism and inosine as potential independent diagnostic biomarkers for major depressive disorder in children and adolescents. Mol Psychiatry 2018.

14. Papakostas GI1, C. C., lovieno N., Folates and S-adenosylmethionine for major depressive disorder. Can J Psychiatry 2012, 57, (7), 406-13.

15. Molle, T.; Moreau, Y.; Clemancey, M.; Forouhar, F.; Ravanat, J. L.; Duraffourg, N.; Fourmond, V.; Latour, J. M.; Gambarelli, S.; Mulliez, E.; Atta, M., Redox Behavior of the S-Adenosylmethionine (SAM)-Binding Fe-S Cluster in Methylthiotransferase Rim0, toward Understanding Dual SAM Activity. 2.997 2016, 55, (41), 5798-5808.

16. Kalender, B.; Ozdemir, A. C.; Koroglu, G., Association of depression with markers of nutrition and inflammation in chronic kidney disease and end-stage renal disease. Nephron Clin Pract 2006, 102, (3-4), c115-21.

17. Duranton, F.; Lundin, U.; Gayrard, N.; Mischak, H.; Aparicio, M.; Mourad, G.; Daures, J. P.; Weinberger, K. M.; Argiles, A., Plasma and Urinary Amino Acid Metabolomic Profiling in Patients with Different Levels of Kidney Function. 5.835 2013, 9, (1), 37-45. 
18. Brocca, A.; Virzì, G. M.; de Cal, M.; Cantaluppi, V.; Ronco, C., Cytotoxic Effects ofp-Cresol in Renal Epithelial Tubular Cells. 1.919 2013, 36, (3-4), 219-225.

19. Pena, M. J.; Lambers Heerspink, H. J.; Hellemons, M. E.; Friedrich, T.; Dallmann, G.; Lajer, M.; Bakker, S. J.; Gansevoort, R. T.; Rossing, P.; de Zeeuw, D.; Roscioni, S. S., Urine and plasma metabolites predict the development of diabetic nephropathy in individuals with Type 2 diabetes mellitus. Diabet Med 2014, 31, (9), 1138-47.

20. Benson, J. M.; Shepherd, D. M., Dietary Ligands of the Aryl Hydrocarbon Receptor Induce AntiInflammatory and Immunoregulatory Effects on Murine Dendritic Cells. 4.181 2011, 124, (2), 327338.

21. Goldstein, L. E.; Leopold, M. C.; Huang, X.; Atwood, C. S.; Saunders, A. J.; Hartshorn, M.; Lim, J. T.; Faget, K. Y.; Muffat, J. A.; Scarpa, R. C.; Chylack, L. T., Jr.; Bowden, E. F.; Tanzi, R. E.; Bush, A. I., 3Hydroxykynurenine and 3-hydroxyanthranilic acid generate hydrogen peroxide and promote alphacrystallin cross-linking by metal ion reduction. 2.997 2000, 39, (24), 7266-75.

22. Felger, J. C.; Li, Z.; Haroon, E.; Woolwine, B. J.; Jung, M. Y.; Hu, X.; Miller, A. H., Inflammation is associated with decreased functional connectivity within corticostriatal reward circuitry in depression. 11.640 2015, 21, (10), 1358-1365.

23. Balta, S.; Demırkol, S.; Kucuk, U., The platelet lymphocyte ratio may be useful inflammatory indicator in clinical practice. $1.2372013, \mathrm{n} / \mathrm{a}-\mathrm{n} / \mathrm{a}$.

24. Turkmen, K.; Guney, l.; Yerlikaya, F. H.; Tonbul, H. Z., The Relationship Between Neutrophil-toLymphocyte Ratio and Inflammation in End-Stage Renal Disease Patients. 1.440 2011, 34, (2), 155159.

25. Birken, D. L.; Oldendorf, W. H., N-acetyl-L-aspartic acid: a literature review of a compound prominent in 1 H-NMR spectroscopic studies of brain. Neurosci Biobehav Rev 1989, 13, (1), 23-31.

26. Tsai, G.; Coyle, J. T., N-acetylaspartate in neuropsychiatric disorders. Prog Neurobio/ 1995, 46, (5), $531-40$.

27. Coppen A, S. D., Malleson A, Eccleston E, Gundy G. , Tryptamine metabolism in depression. Br J Psychiatry 1965, 993-8.

28. Mayr, J. A.; Freisinger, P.; Schlachter, K.; Rolinski, B.; Zimmermann, F. A.; Scheffner, T.; Haack, T. B.; Koch, J.; Ahting, U.; Prokisch, H.; Sperl, W., Thiamine pyrophosphokinase deficiency in encephalopathic children with defects in the pyruvate oxidation pathway. Am J Hum Genet 2011, 89, (6), 806-12.

29. Gibson, G. E.; Blass, J. P., Thiamine-dependent processes and treatment strategies in neurodegeneration. 6.530 2007, 9, (10), 1605-19.

30. Ke, Z. J.; Gibson, G. E., Selective response of various brain cell types during neurodegeneration induced by mild impairment of oxidative metabolism. Neurochem Int 2004, 45, (2-3), 361-9.

31. Zhang, G.; Ding, H.; Chen, H.; Ye, X.; Li, H.; Lin, X.; Ke, Z., Thiamine nutritional status and depressive symptoms are inversely associated among older Chinese adults. J Nutr 2013, 143, (1), 53-8. 
32. Paul, I. A.; Skolnick, P., Glutamate and depression: clinical and preclinical studies. Ann N Y Acad Sci 2003, 1003, 250-72.

33. Horrobin, D. F., Phospholipid metabolism and depression: the possible roles of phospholipase A 2 and coenzyme A-independent transacylase. Human Psychopharmacology Clinical \& Experimental 2001, 16, (1), 45-52.

34. Wang, L.; Radu, C. G.; Yang, L. V.; Bentolila, L. A.; Riedinger, M.; Witte, O. N., Lysophosphatidylcholineinduced surface redistribution regulates signaling of the murine $G$ protein-coupled receptor $G 2 A$. 3.512 2005, 16, (5), 2234.

35. Van Den Pol, A. N.; Wuarin, J.-P.; Dudek, F. E., Glutamate, the dominant excitatory transmitter in neuroendocrine regulation. Science 1990, 250, (4985), 1276-1278.

36. Akbarian, S.; Huntsman, M. M.; Kim, J. J.; Tafazzoli, A.; Potkin, S. G.; Bunney, W. E., Jr.; Jones, E. G., GABAA receptor subunit gene expression in human prefrontal cortex: comparison of schizophrenics and controls. Cereb Cortex 1995, 5, (6), 550-60.

37. Gunnersen, D.; Haley, B., Detection of glutamine synthetase in the cerebrospinal fluid of Alzheimer diseased patients: a potential diagnostic biochemical marker. Proc Natl Acad Sci U S A 1992, 89, (24), 11949-53.

38. Cryan, J. F.; Kelly, P. H.; Neijt, H. C.; Sansig, G.; Flor, P. J.; van Der Putten, H., Antidepressant and anxiolytic-like effects in mice lacking the group III metabotropic glutamate receptor mGluR7. European Journal of Neuroscience 2003, 17, (11), 2409-2417.

39. Hamilton, M., A rating scale for depression. J Neurol Neurosurg Psychiatry 1960, 23, (1), 56-62.

40. Wishart, D. S.; Knox, C.; An, C. G.; Eisner, R.; Young, N.; Gautam, B.; Hau, D. D.; Psychogios, N.; Dong, E.; Bouatra, S., HMDB: a knowledgebase for the human metabolome. 11.561 2009, 37, (Database issue), 603-10.

41. Newgard, C. B.; An, J.; Bain, J. R.; Muehlbauer, M. J.; Stevens, R. D.; Lien, L. F.; Haqq, A. M.; Shah, S. H.; Arlotto, M.; Slentz, C. A.; Rochon, J.; Gallup, D.; Ilkayeva, O.; Wenner, B. R.; Yancy, W. S., Jr.; Eisenson, H.; Musante, G.; Surwit, R. S.; Millington, D. S.; Butler, M. D.; Svetkey, L. P., A branched-chain amino acid-related metabolic signature that differentiates obese and lean humans and contributes to insulin resistance. Cell Metab 2009, 9, (4), 311-26.

42. Wang, T. J.; Larson, M. G.; Vasan, R. S.; Cheng, S.; Rhee, E. P.; McCabe, E.; Lewis, G. D.; Fox, C. S.; Jacques, P. F.; Fernandez, C.; O'Donnell, C. J.; Carr, S. A.; Mootha, V. K.; Florez, J. C.; Souza, A.; Melander, O.; Clish, C. B.; Gerszten, R. E., Metabolite profiles and the risk of developing diabetes. Nat Med 2011, 17, (4), 448-53.

43. Westerhuis, J. A.; van Velzen, E. J.; Hoefsloot, H. C.; Smilde, A. K., Multivariate paired data analysis: multilevel PLSDA versus OPLSDA. 3.511 2010, 6, (1), 119-128.

\section{Tables}


Table 1. Demographic and clinical characteristics of the three groups

\begin{tabular}{|c|c|c|c|}
\hline Indicatiors & $\begin{array}{l}\text { Control } \\
\text { group }\end{array}$ & $\begin{array}{c}\text { ESRD without depression } \\
\text { group }\end{array}$ & $\begin{array}{c}\text { ESRD with depression } \\
\text { group }\end{array}$ \\
\hline Age (year) & $57.00 \pm 11.51$ & $50.18 \pm 10.65$ & $50.82 \pm 13.45$ \\
\hline Hypertension history (month) & $0.00 \pm 0.00$ & $73.82 \pm 61.47^{* *}$ & $66.76 \pm 85.80^{*}$ \\
\hline Diabetes history (month) & $0.00 \pm 0.00$ & $52.24 \pm 88.69$ & $43.06 \pm 98.23$ \\
\hline $\begin{array}{l}\text { History of nephropathy } \\
\text { (month) }\end{array}$ & $0.00 \pm 0.00$ & $42.00 \pm 38.29^{* *}$ & $30.71 \pm 43.41^{*}$ \\
\hline Heart disease history (month) & $0.00 \pm 0.00$ & $0.29 \pm 1.21$ & $7.76 \pm 16.40$ \\
\hline SBP $(\mathrm{mmHg})$ & $126.33 \pm 4.50$ & $163.53 \pm 23.15^{* *}$ & $171.12 \pm 33.06^{* *}$ \\
\hline DBP (mmHg) & $80.33 \pm 2.67$ & $94.24 \pm 9.75^{* *}$ & $101.59 \pm 16.19^{* *}$ \\
\hline $\begin{array}{l}\text { Dialysis frequency } \\
\text { (times/week) }\end{array}$ & $0.00 \pm 0.00$ & $2.53 \pm 1.01^{* *}$ & $2.12 \pm 1.11^{* *}$ \\
\hline WBC $\left(\times 10^{9} / \mathrm{L}\right)$ & $5.79 \pm 1.55$ & $7.22 \pm 2.53$ & $6.62 \pm 3.48$ \\
\hline $\mathrm{N}(\%)$ & $58.56 \pm 8.17$ & $69.23 \pm 9.82^{*}$ & $78.24 \pm 11.20^{* *} \triangle$ \\
\hline $\mathrm{RBC}\left(\times 10^{12} / \mathrm{L}\right)$ & $4.67 \pm 0.35$ & $3.73 \pm 0.63^{* *}$ & $3.17 \pm 1.13^{* *}$ \\
\hline $\mathrm{Hb}(\mathrm{g} / \mathrm{L})$ & $142.50 \pm 13.36$ & $109.71 \pm 17.09^{* *}$ & $92.29 \pm 25.84^{* *}$ \\
\hline$\Gamma \mathrm{C}(\mathrm{mmol} / \mathrm{L})$ & $4.72 \pm 0.92$ & $3.25 \pm 1.57^{*}$ & $4.30 \pm 1.68$ \\
\hline TG $(\mathrm{mmol} / \mathrm{L})$ & $1.25 \pm 0.68$ & $3.13 \pm 1.50^{* *}$ & $2.02 \pm 1.46$ \\
\hline Albumin $(g / L)$ & $44.88 \pm 3.66$ & $38.39 \pm 2.92^{* *}$ & $124.34 \pm 280.64$ \\
\hline $\operatorname{Cr}(\mu \mathrm{mol} / \mathrm{L})$ & $59.10 \pm 12.43$ & $985.87 \pm 342.62^{* *}$ & $915.14 \pm 213.44^{* *}$ \\
\hline BUN (mmol/L) & $5.38 \pm 1.43$ & $21.37 \pm 5.85^{* *}$ & $28.72 \pm 7.23^{* *} \Delta \triangle$ \\
\hline $\mathrm{K}^{+}(\mathrm{mmol} / \mathrm{L})$ & $4.04 \pm 0.51$ & $4.78 \pm 0.56^{* *}$ & $4.67 \pm 0.81^{*}$ \\
\hline Phosphoru (mmol/L) & $1.09 \pm 0.12$ & $1.82 \pm 0.47^{* *}$ & $1.97 \pm 0.82^{* *}$ \\
\hline $\mathrm{Ca}^{2+}(\mathrm{mmol} / \mathrm{L})$ & $2.32 \pm 0.15$ & $2.15 \pm 0.32$ & $2.07 \pm 0.33^{*}$ \\
\hline Anxiety scores & $4.33 \pm 5.66$ & $7.41 \pm 3.40$ & $21.29 \pm 4.91^{* *} \triangle \triangle$ \\
\hline Depression scores & $3.75 \pm 3.11$ & $9.00 \pm 3.06^{* *}$ & $22.88 \pm 4.20^{* *} \Delta \triangle$ \\
\hline
\end{tabular}

SBP: systolic blood pressure; DBP: diastolic blood pressure; WBC: white blood cells; N: neutrophils; RBC: red blood cells; Hb: hemoglobin; TC: cholesterol; TG: triglycerides; Cr: serum creatinine; BUN: blood urea nitrogen. Compared with the control group, ${ }^{*}: p<0.05,{ }^{* *} p<0.01$; compared with ESRD with depression group, ${ }^{\Delta}: p<$ $0.05,{ }^{\Delta \Delta}: p<0.01$

Table 2. Characteristic compounds of ESRD without depression group vs. ESRD with depression group 


\begin{tabular}{|c|c|c|c|c|c|}
\hline Compound name & $\mathrm{ESI}^{+/-}$ & FC & raw.pval & vip & Trend \\
\hline 5'-Deoxy-5-fluorocytidine & + & 5.444 & 0.0263 & 2.0509 & $\uparrow$ \\
\hline Dehydrogenated ticlopidine & + & 31.16 & 0.019107 & 2.1539 & $\uparrow$ \\
\hline N-Desmethylpromazine & + & 0.0012086 & 0.015404 & 2.2204 & $\downarrow$ \\
\hline Leucyl-phenylalanine & + & 0.0397 & 0.036056 & 1.9432 & $\downarrow$ \\
\hline Cladribine & + & 7.958 & 0.048751 & 1.8341 & $\uparrow$ \\
\hline Pregnenolone & + & 0.0029882 & 0.045939 & 1.8561 & $\downarrow$ \\
\hline Bevantolol & + & 0.03225 & 0.046476 & 1.8518 & $\downarrow$ \\
\hline Tetracosanoic acid & + & 9619 & 0.022889 & 2.0964 & $\uparrow$ \\
\hline 6-Thioguanosine monophosphate & + & 23.301 & 0.049064 & 1.8318 & $\uparrow$ \\
\hline Farnesyl pyrophosphate & + & 6.3943 & 0.038448 & 1.9205 & $\uparrow$ \\
\hline Alfuzosin & + & 0.018852 & 0.006956 & 2.4468 & $\downarrow$ \\
\hline Cephapirin & + & 15.773 & 0.022201 & 2.1062 & $\uparrow$ \\
\hline Thiamine pyrophosphate & + & 8.7289 & 0.021436 & 2.1175 & $\uparrow$ \\
\hline Cabergoline & + & 0.011239 & 0.023683 & 2.0853 & $\downarrow$ \\
\hline Almitrine & + & 0.098922 & 0.046332 & 1.853 & $\downarrow$ \\
\hline Probucol & + & 0.026526 & 0.021199 & 2.121 & $\downarrow$ \\
\hline LysoPC(22:4(7Z,10Z,13Z,16Z)) & + & 0.040511 & 0.045457 & 1.86 & $\downarrow$ \\
\hline PA $(16: 0 / 18: 2(9 Z, 12 Z))$ & + & 0.022735 & 0.003310 & 2.6358 & $\downarrow$ \\
\hline PG $(18: 0 / 18: 1(11 Z))$ & + & 0.0014305 & 0.041451 & 1.8936 & $\downarrow$ \\
\hline CL (i-14:0/i-12:0/i-14:0/18:2(9Z,11Z)) & + & 0.067084 & 0.007024 & 2.4442 & $\downarrow$ \\
\hline Phosphoric acid & - & 0.04634 & 0.023768 & 1.893 & $\downarrow$ \\
\hline 4-Methylcatechol & - & 0.041521 & 0.029016 & 1.8329 & $\downarrow$ \\
\hline Dihydrothymine & - & 29.157 & 0.000168 & 2.9428 & $\uparrow$ \\
\hline 3-Methyl-2-oxovaleric acid & - & 0.092461 & 0.006130 & 2.2528 & $\downarrow$ \\
\hline Urocanic acid & - & 0.096113 & 0.048671 & 1.6664 & $\downarrow$ \\
\hline 5-ethyl-5-methyl-2,4-oxazolidinedione & - & 4.65E-09 & 0.036618 & 1.76 & $\downarrow$ \\
\hline L-Glutamic acid & - & 24.417 & 0.000410 & 2.7978 & $\uparrow$ \\
\hline Gentisic acid & - & 2.57E-09 & 0.046875 & 1.6791 & $\downarrow$ \\
\hline 2,5-Furandicarboxylic acid & - & 7.19E-09 & 0.032056 & 1.8021 & $\downarrow$ \\
\hline L-3-Phenyllactic acid & - & 7.2911 & 0.048732 & 1.666 & $\uparrow$ \\
\hline Uric acid & - & $1.42 \mathrm{E}-09$ & 0.040158 & 1.7302 & $\downarrow$ \\
\hline N-Acetyl-L-aspartic acid & - & 0.023715 & 0.004395 & 2.3306 & $\downarrow$ \\
\hline Serotonin & - & 0.022792 & 0.017415 & 1.9826 & $\downarrow$ \\
\hline Vanylglycol & - & 0.0047169 & 0.027679 & 1.8473 & $\downarrow$ \\
\hline 2-Phosphoglyceric acid & - & 0.056671 & 0.002838 & 2.4274 & $\downarrow$ \\
\hline Homocitrulline & - & 6.0653 & 0.002780 & 2.4319 & $\uparrow$ \\
\hline Hydroxyphenylacetylglycine & - & 6.8042 & 0.007710 & 2.1971 & $\uparrow$ \\
\hline 1-Hydroxylorcaserin & - & 1.32E-09 & 0.026271 & 1.8631 & $\downarrow$ \\
\hline Propenoylcarnitine & - & 2.18E-09 & 0.042833 & 1.7091 & $\downarrow$ \\
\hline cyclic 6-Hydroxymelatonin & - & 32.34 & 0.000443 & 2.7843 & $\uparrow$ \\
\hline Desacetyl-nitazoxanide & - & 10.564 & 0.01959 & 1.9492 & $\uparrow$ \\
\hline Deoxyartemsinin & - & 17.749 & 0.000381 & 2.8099 & $\uparrow$ \\
\hline Malaoxon & - & 0.056423 & 0.015184 & 2.0206 & $\downarrow$ \\
\hline 5 -HEPE & - & 0.074764 & 0.019045 & 1.9573 & $\downarrow$ \\
\hline 15(S)-Hydroxyeicosatrienoic acid & - & 0.019982 & 0.023266 & 1.8993 & $\downarrow$ \\
\hline 5'-O-Desmethyl omeprazole & - & 0.040628 & 0.000461 & 2.7776 & $\downarrow$ \\
\hline 17-HDoHE & - & 0.086085 & 0.012912 & 2.0645 & $\downarrow$ \\
\hline $\begin{array}{l}\text { 5-( } 4 \text { '-Hydroxyphenyl)-gamma-valerolactone-4'-O- } \\
\text { glucuronide }\end{array}$ & - & 8.9677 & 0.023437 & 1.8971 & $\uparrow$ \\
\hline Hydromorphone-3-sulphate & - & 5.4727 & 0.045925 & 1.686 & $\uparrow$ \\
\hline 6-Thioxanthine 5'-monophosphate & - & 0.082069 & 0.007364 & 2.2084 & $\downarrow$ \\
\hline Hydroxylansoprazole & - & 8.92E-09 & 0.046771 & 1.6799 & $\downarrow$ \\
\hline Eletriptan N-oxide & - & 0.060055 & 0.02196 & 1.9162 & $\downarrow$ \\
\hline Carboxycelecoxib & - & 0.060337 & 0.033844 & 1.7851 & $\downarrow$ \\
\hline Zileuton O-glucuronide & - & 2.53E-09 & 0.014994 & 2.0241 & $\downarrow$ \\
\hline Hesperetin 3'-O-glucuronide & - & 7.9491 & 0.037187 & 1.7551 & $\uparrow$ \\
\hline DG (14:0/16:1(9Z)/0:0) & - & 7.0082 & 0.008436 & 2.1747 & $\uparrow$ \\
\hline Enkephalin L & - & 10.077 & 0.035126 & 1.7733 & $\uparrow$ \\
\hline
\end{tabular}


Table 3. Different metabolite Pathway in ESRD without depression group vs. ESRD with depression group

\begin{tabular}{|c|c|c|c|c|c|c|c|}
\hline & Total & Expected & Hits & Raw $p$ & $\begin{array}{l}\text { Holm } \\
\text { adjust }\end{array}$ & DR & Impact \\
\hline $\begin{array}{l}\text { Alanine, aspartate and glutamate } \\
\text { metabolism }\end{array}$ & 24 & 0.2393 & 2 & 0.02301 & 1 & 1 & 0.17664 \\
\hline Tyrosine metabolism & 76 & 0.75779 & 3 & 0.038024 & 1 & 1 & 0.01825 \\
\hline Glycerophospholipid metabolism & 39 & 0.38887 & 2 & 0.056434 & 1 & 1 & 0.10429 \\
\hline Histidine metabolism & 44 & 0.43872 & 2 & 0.069917 & 1 & 1 & 0.06816 \\
\hline D-Glutamine and D-glutamate metabolism & 11 & 0.10968 & 1 & 0.10458 & 1 & 1 & 0.1123 \\
\hline Citrate cycle (TCA cycle) & 20 & 0.19942 & 1 & 0.18227 & 1 & 1 & 0.04844 \\
\hline Thiamine metabolism & 24 & 0.2393 & 1 & 0.21468 & 1 & 1 & 0.02865 \\
\hline Glycolysis or Gluconeogenesis & 31 & 0.3091 & 1 & 0.26847 & 1 & 1 & 0.00068 \\
\hline Pyruvate metabolism & 32 & 0.31907 & 1 & 0.27586 & 1 & 1 & 0.00459 \\
\hline Glycerolipid metabolism & 32 & 0.31907 & 1 & 0.27586 & 1 & 1 & 0.01247 \\
\hline Terpenoid backbone biosynthesis & 33 & 0.32904 & 1 & 0.28317 & 1 & 1 & 0.05141 \\
\hline Glutathione metabolism & 38 & 0.37889 & 1 & 0.31871 & 1 & 1 & 0.01095 \\
\hline Valine, leucine and isoleucine degradation & 40 & 0.39884 & 1 & 0.33245 & 1 & 1 & 0.03781 \\
\hline Pyrimidine metabolism & 60 & 0.59826 & 1 & 0.45599 & 1 & 1 & 0.01492 \\
\hline Aminoacyl-tRNA biosynthesis & 75 & 0.74782 & 1 & 0.53393 & 1 & 1 & 0.05634 \\
\hline Arginine and proline metabolism & 77 & 0.76776 & 1 & 0.54348 & 1 & 1 & 0.03582 \\
\hline Tryptophan metabolism & 79 & 0.7877 & 1 & 0.55284 & 1 & 1 & 0.05734 \\
\hline Purine metabolism & 92 & 0.91732 & 1 & 0.60933 & 1 & 1 & 0.00969 \\
\hline Steroid hormone biosynthesis & 99 & 0.98712 & 1 & 0.63685 & 1 & 1 & 0.02542 \\
\hline
\end{tabular}

Table 4 The metabolites for the diagnosis of ESRD with depression (AUC $\geq 0.90$ ) 


\begin{tabular}{lllll}
\hline Compound & AUC & TPR & FPR & 95\% CI \\
\hline 5-ethyl-5-methyl-2,4-oxazolidinedione & 0.934 & 0.9 & 0.8 & $0.834-0.986$ \\
Gentisic acid & 0.965 & 0.9 & 0.9 & $0.896-1$ \\
2,5-Furandicarboxylic acid & 0.931 & 0.9 & 0.8 & $0.829-0.986$ \\
Uric acid & 0.931 & 0.9 & 0.8 & $0.834-0.99$ \\
Serotonin & 0.931 & 0.9 & 0.9 & $0.822-0.986$ \\
2-Phosphoglyceric acid & 0.924 & 0.8 & 0.9 & $0.811-0.986$ \\
N-Desmethylpromazine & 0.965 & 0.9 & 0.9 & $0.9-1$ \\
Leucyl-phenylalanine & 0.938 & 0.9 & 0.9 & $0.834-0.99$ \\
Propenoyl carnitine & 0.934 & 0.9 & 0.8 & $0.832-0.99$ \\
Malaoxon & 0.927 & 0.9 & 0.8 & $0.803-0.99$ \\
Pregnenolone & 0.976 & 0.9 & 0.9 & $0.917-1$ \\
Bevantolol & 0.948 & 0.9 & 0.9 & $0.861-1$ \\
6-Thioxanthine 5'-monophosphate & 0.924 & 0.9 & 0.9 & $0.789-1$ \\
Hydroxyl ansoprazole & 0.924 & 0.9 & 0.8 & $0.824-0.986$ \\
Alfuzosin & 0.931 & 0.8 & 0.9 & $0.823-0.986$ \\
Zileuton O-glucuronide & 0.958 & 0.9 & 0.9 & $0.867-1$ \\
Cabergoline & 0.952 & 0.9 & 0.8 & $0.849-0.997$ \\
Probucol & 0.931 & 0.9 & 0.8 & $0.815-0.986$ \\
PA (16:0/18:2(9Z,12Z)) & 0.945 & 0.9 & 0.9 & $0.839-1$ \\
PG (18:0/18:1(11Z)) & 0.9 & 0.9 & $0.875-1$ \\
\hline
\end{tabular}

TPR: True positive rate; FPR: False positive rate; 95\% CI: 95\% Confidence interval 


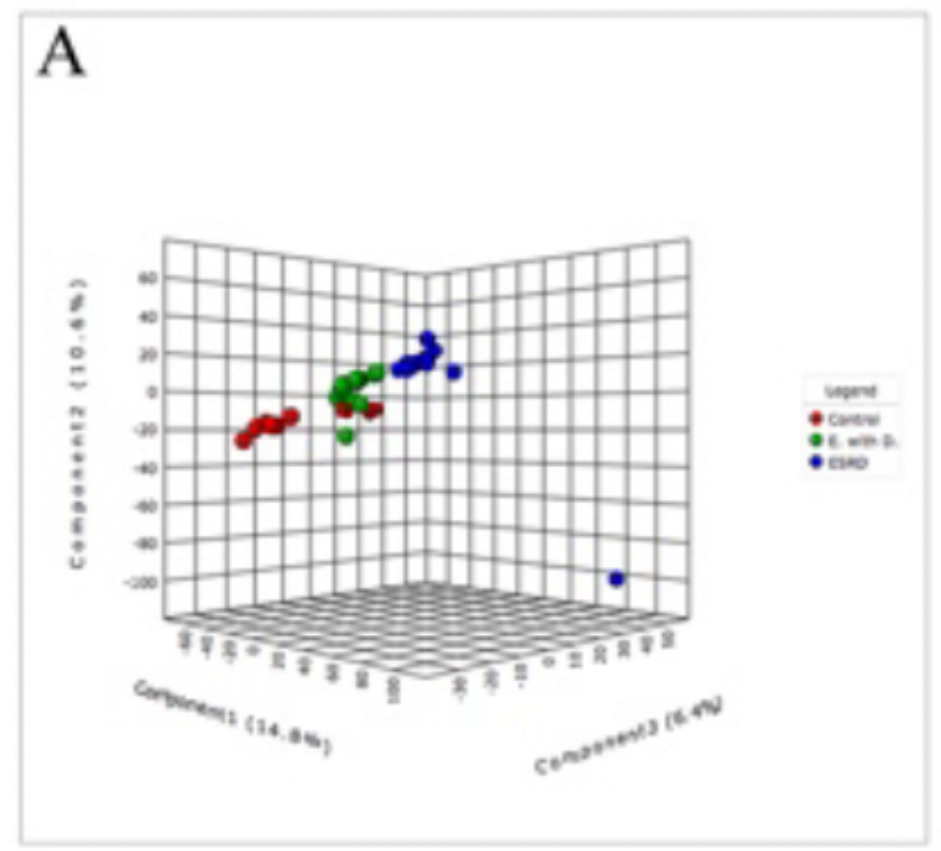

\section{B}
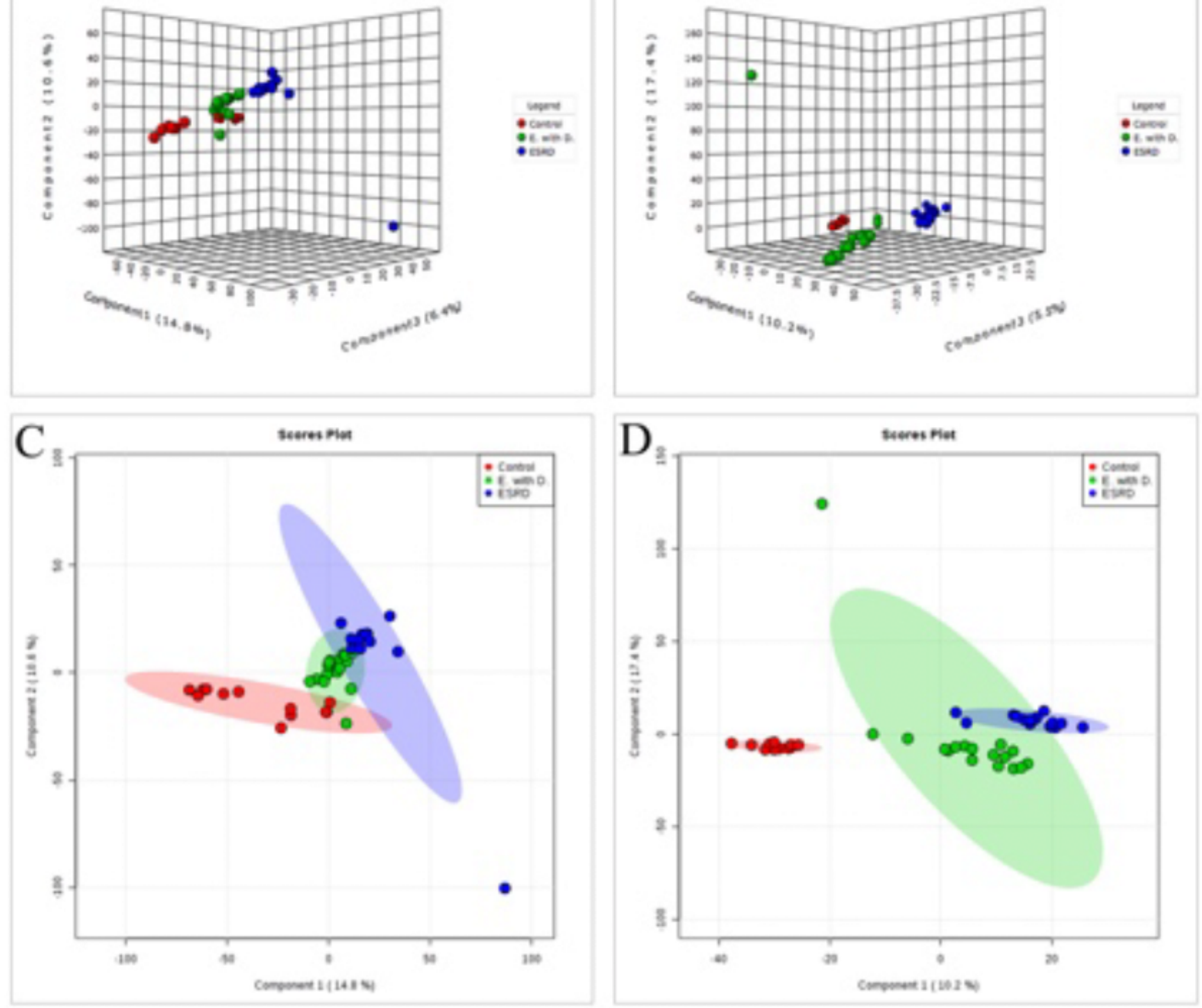

\section{Figure 1}

The score plots of the three groups of PLS-DA model in positive ion mode (A, C) and negative ion mode $(B, D)$ 


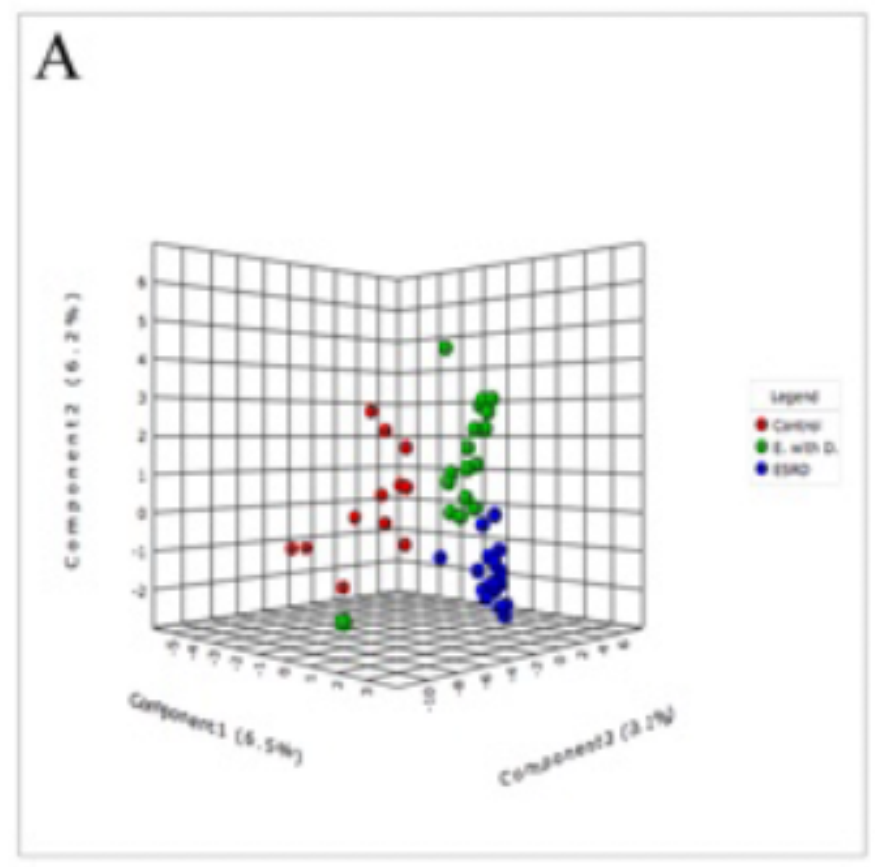

\section{B}
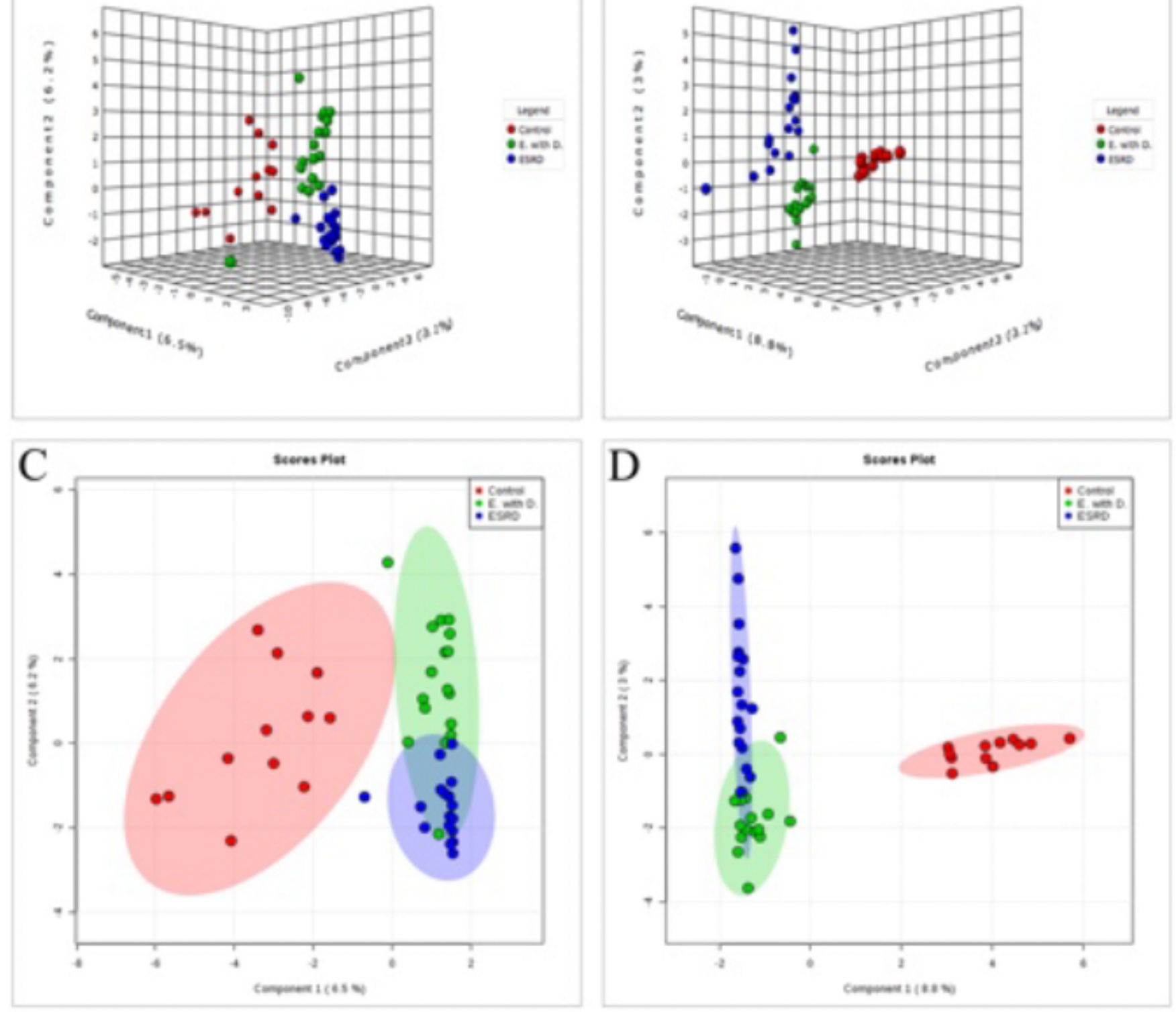

Figure 2

The score plots of the three groups of OPLS-DA model in positive ion mode $(A, C)$ and negative ion mode $(B, D)$ 

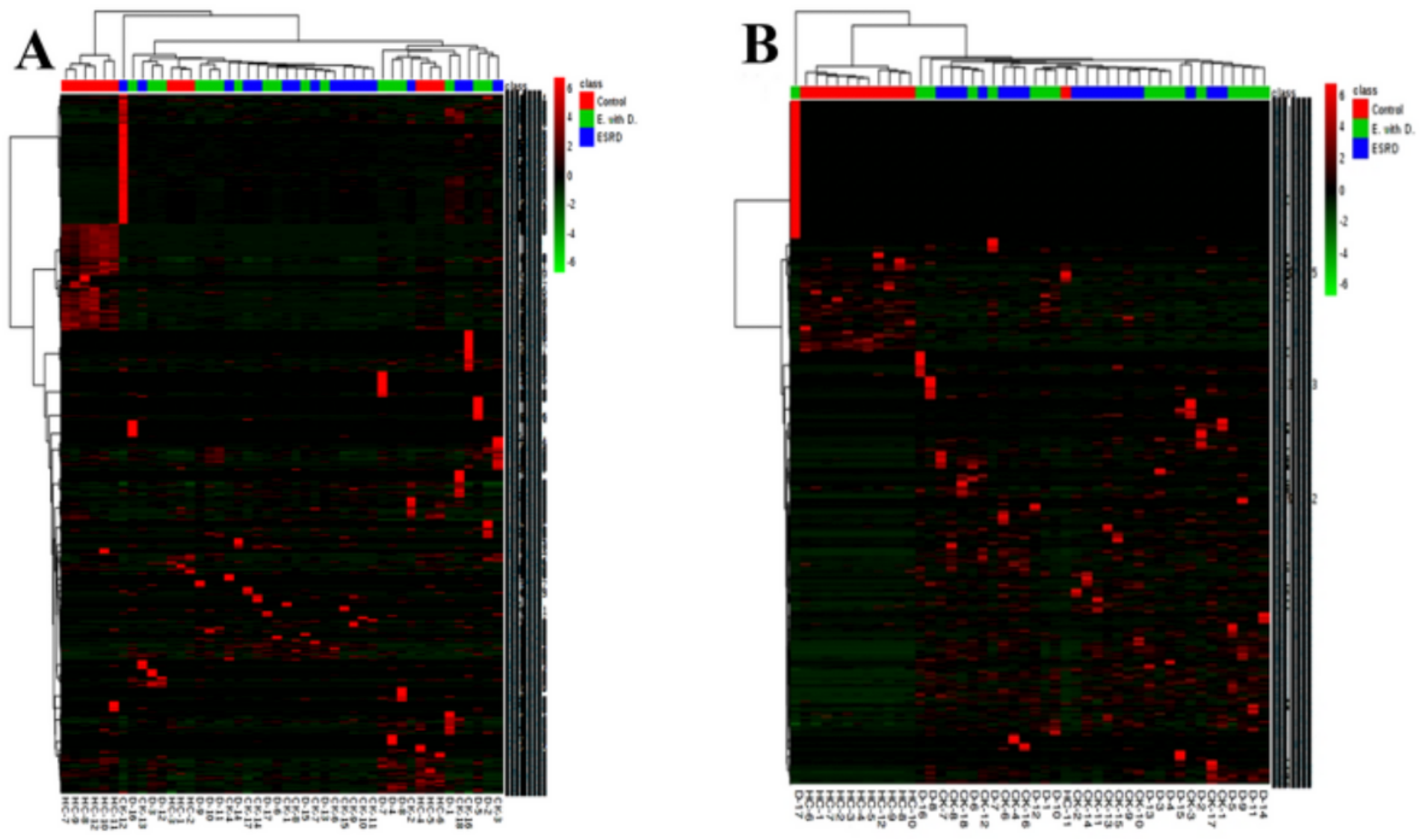

\section{Figure 3}

Thermogram of the relative content of differential metabolites: $A$ is in Positive ion mode, $B$ is in negative ion mode 


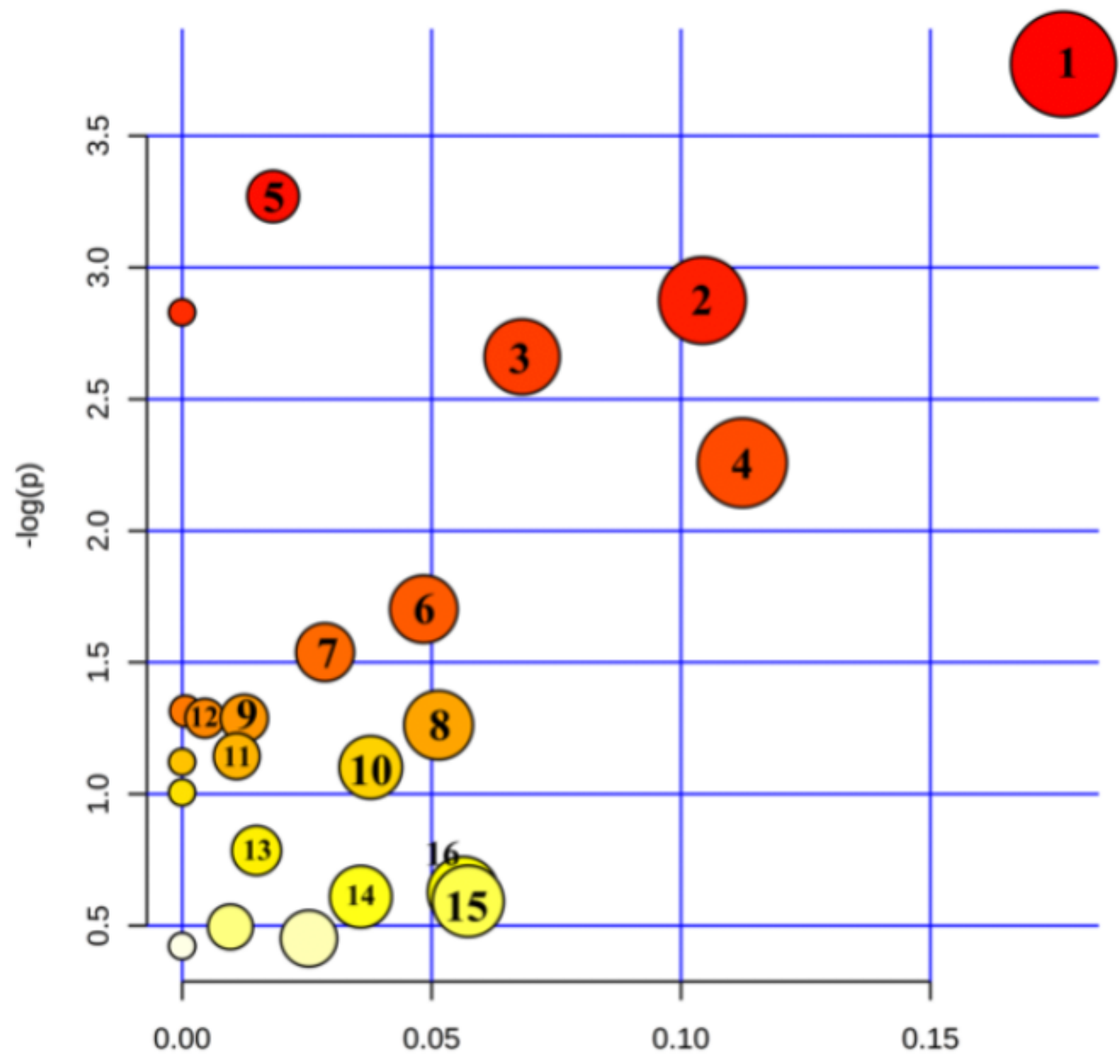

Pathway Impact

\section{Figure 4}

Scores of metabolite pathways involved by differential metabolites in ESRD without depression group vs. ESRD with depression group. The abscissa is the importance value of the compound in the pathway, and the ordinate is the negative logarithm of the $p$-value log with a base of 10 . The closer to the upper right corner, the more significant the enrichment represents and the more important the role of the compound plays in the pathway. 1: Alanine, aspartate and glutamate metabolism; 2: Glycerophospholipid metabolism, 3: Histidine metabolism; 4: D-Glutamine and D-glutamate metabolism; 5: Tyrosine metabolism; 6: Citrate cycle (TCA cycle); 7: Thiamine metabolism; 8: Terpenoid backbone biosynthesis; 9: Valine, leucine and isoleucine degradation; 10: Glycerophospholipid metabolism; 11: Glutathione 
metabolism; 12: Pyruvate metabolism; 13: Pyrimidine metabolism; 14: Arginine and proline metabolism; 15: Tryptophan metabolism; 16: Aminoacyl-tRNA biosynthesis

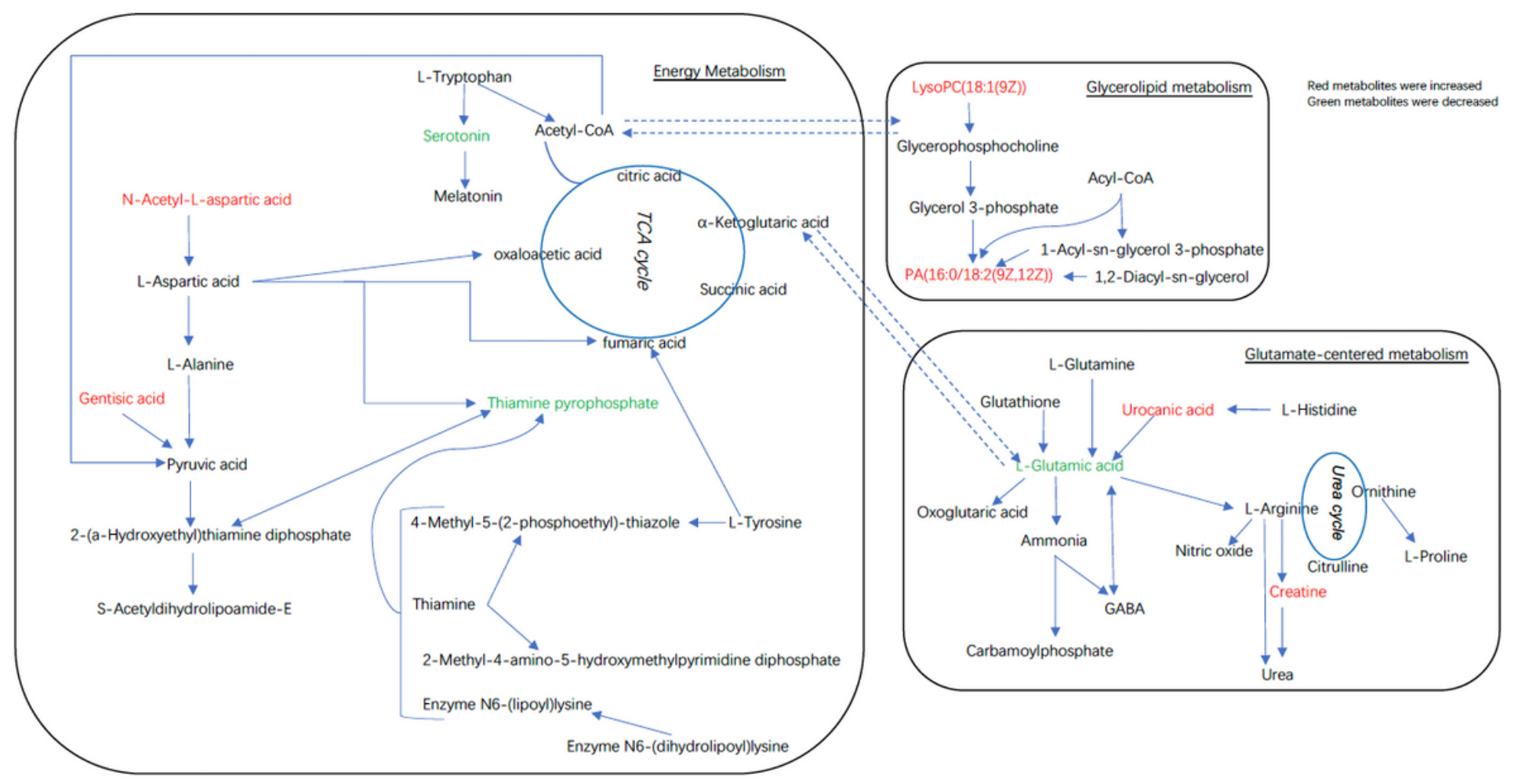

\section{Figure 5}

Biochemical transformation of differential metabolites Note: ESRD patients with depression have increased red metabolites and decreased green metabolites compared with ESRD patients without depression. 